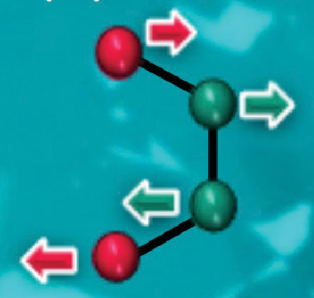

\title{
Faraday Discussions
}

Volume: 227

Chemistry of

2-Dimensional Materials: Beyond Graphene 


\title{
PAPER
}

\section{Raman spectroscopy of GaSe and InSe post-transition metal chalcogenides layers $\uparrow$}

\author{
Maciej R. Molas, (D) a Anastasia V. Tyurnina, ${ }^{\text {bcd }}$ Viktor Zólyomi, ${ }^{\text {bc }}$ \\ Anna K. Ott, ${ }^{\text {e }}$ Daniel J. Terry, ${ }^{\text {bc }}$ Matthew J. Hamer, ${ }^{\text {bc }}$ Celal Yelgel, ${ }^{c}$ \\ Adam Babiński, (D) a Albert G. Nasibulin, ${ }^{d}$ Andrea C. Ferrari, ${ }^{\text {*e }}$ \\ Vladimir I. Fal'kobcf and Roman Gorbachev ${ }^{\text {bcf }}$
}

Received 12th January 2020, Accepted 22nd January 2020

DOI: $10.1039 / \mathrm{dOfd00007h}$

III-VI post-transition metal chalcogenides (InSe and GaSe) are a new class of layered semiconductors, which feature a strong variation of size and type of their band gaps as a function of number of layers $(N)$. Here, we investigate exfoliated layers of InSe and GaSe ranging from bulk crystals down to monolayer, encapsulated in hexagonal boron nitride, using Raman spectroscopy. We present the $N$-dependence of both intralayer vibrations within each atomic layer, as well as of the interlayer shear and layer breathing modes. A linear chain model can be used to describe the evolution of the peak positions as a function of $N$, consistent with first principles calculations.

\section{Introduction}

Layered materials (LMs) are at the centre of an ever-increasing research effort spanning across a multitude of scientific disciplines. ${ }^{1}$ In addition to widely popular semiconducting transition metal dichalcogenides (TMDs), such as $\mathrm{MoS}_{2}$, $\mathrm{MoSe}_{2}, \mathrm{MoTe}_{2}, \mathrm{WS}_{2}$ and $\mathrm{WSe}_{2}$, a new class of LMs, semiconducting posttransition metal chalcogenides (PTMCs), are increasingly studied. ${ }^{2-6}$ These materials feature a large variation of the optical bandgap with number of layers, $N$ : from $1.25 \mathrm{eV}$ in bulk to $2.8 \mathrm{eV}$ in monolayer (1L) InSe $\mathrm{I}^{3,4,6}$ and from $2.0 \mathrm{eV}$ (bulk)

anstitute of Experimental Physics, Faculty of Physics, University of Warsaw, Pasteura 5, 02-093 Warszawa, Poland

${ }^{b}$ School of Physics and Astronomy, University of Manchester, Oxford Road, M13 9PL, UK

${ }^{c}$ National Graphene Institute, University of Manchester, Booth St E, Manchester, M13 9PL, UK

${ }^{d}$ Skolkovo Institute of Science and Technology, Nobel St. 3, 143026 Moscow, Russia

${ }^{e}$ Cambridge Graphene Centre, University of Cambridge, $9 \mathrm{JJ}$ Thomson Avenue, Cambridge, CB3 OFA, UK. E-mail: acf26@eng.cam.ac.uk

${ }^{{ }^{f} H e n r y}$ Royce Institute, University of Manchester, Oxford Road, Manchester, M13 9PL, UK

$\dagger$ Electronic supplementary information (ESI) available: S1: selection rules for $\mathrm{E}^{\prime}$ and $\mathrm{E}^{\prime \prime}$ phonons. S2: $\Gamma$-point frequencies in FL-GaSe and InSe. S3: resonance effects. See DOI: 10.1039/d0fd00007h 
to $2.4 \mathrm{eV}$ (bilayer, 2L) in GaSe. ${ }^{5}$ Furthermore, the band-gap evolves from being quasi-direct for $1 \mathrm{~L}$ to direct in bulk. These materials also feature outstanding electronic transport properties, e.g. in InSe mobilities reach up to $10^{3} \mathrm{~cm}^{2} \mathrm{~V}^{-1} \mathrm{~s}^{-1}$ and $10^{4} \mathrm{~cm}^{2} \mathrm{~V}^{-1} \mathrm{~s}^{-1}$ at room and liquid-helium temperatures ${ }^{4}$, as well as onedimensional quantization of electrons by electrostatic gating, ${ }^{7}$ and unusual photoluminescence, polarized primarily out of the basal plane. ${ }^{6}$ They can be combined into PTMC/PTMC or PTMC/TMD layered materials heterostructures (LMHs), with type-II band alignment, allowing direct optical transitions in reciprocal space, ${ }^{8}$ and offering an even larger selection of emission energies, ${ }^{5}$ with potential for novel optoelectronic applications in a broad spectral range, from far infra-red to violet.

GaSe and InSe crystals are anisotropic LMs comprising covalently bonded layers stacked together by van der Waals forces. Each layer consists of four atomic planes (Se-Ga-Ga-Se or Se-In-In-Se) arranged in a hexagonal atomic lattice, Fig. 1a and b. In bulk, these layers can be stacked in a different orders: a hexagonal $\beta$-structure belonging to the $D_{6 h}^{4}$ space group, a hexagonal $\varepsilon$-structure belonging to the $D_{3 h}^{1}$ space group or a rhombohedral $\gamma$-structure belonging to the $D_{3 v}^{S}$ space group. ${ }^{9}$ However, the most commonly found polytypes are $\varepsilon$-GaSe, with a unit cell containing 8 atoms and two layers thick, ${ }^{5}$ and $\gamma$-InSe, with a unit cell extending over 3 layers, containing 12 atoms. $^{6}$

Raman spectroscopy is the prime non-destructive characterization tool for graphene and LMs. ${ }^{10-13}$ In LMs there are 2 fundamentally different sets of Raman modes. Those coming from the relative motion of atoms within each layer, usually found at high frequencies, ${ }^{\mathbf{1 0}, 11}$ and those due to relative motions of the atomic planes themselves, either parallel to each other (C modes $)^{12}$ or perpendicular (layer breathing modes, LBMs). ${ }^{13}$ For the latter the experimental results can be interpreted using with a simple linear chain model (LCM), whereby each plane is

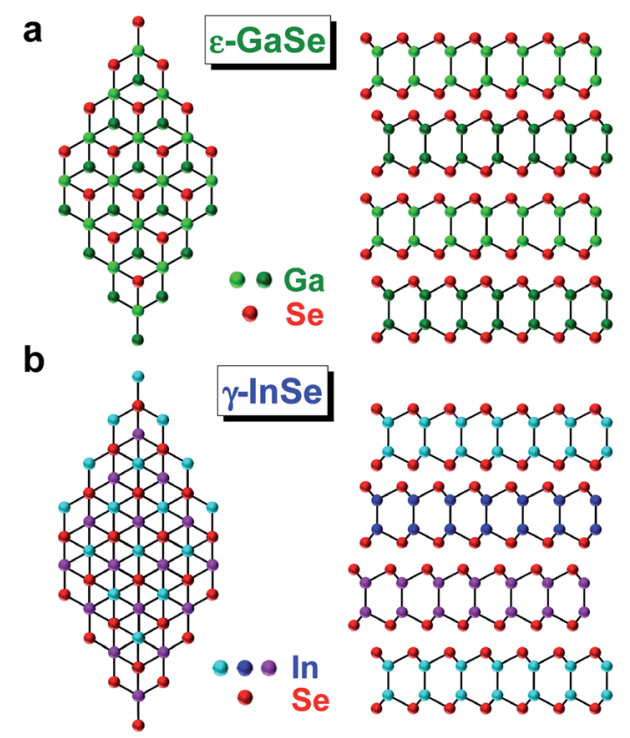

Fig. 1 Atomic structure and stacking order of (a) $\varepsilon$-GaSe and (b) $\gamma$-InSe. 
considered linked to the next one by a spring corresponding to an interlayer force constant per unit area (parallel or perpendicular to the planes for the two types of motion). ${ }^{12,13}$

So far, Raman characterization of PTMCs has been reported on few-layers thick crystals. ${ }^{14-18}$ Due to low signal-to-noise ratio and strong degradation occurring in ambient conditions, ${ }^{19}$ the thinnest PTMCs have not been investigated properly: background noise or incomplete spectra were reported, containing only one prominent Raman feature for $1 \mathrm{~L}$ and $2 \mathrm{~L}$ samples. ${ }^{\mathbf{1 4 - 1 8}}$

Here, we present a Raman study of high crystalline quality InSe and GaSe flakes prepared by mechanical exfoliation, with thicknesses varying from bulk to 1L, encapsulated in hexagonal boron nitride (hBN) in an inert argon environment. We observe the thickness-dependent evolution of both intralayer vibrations as well as C and LB modes, and compare the experimental results with first principles calculations. We demonstrate that Raman spectroscopy can be used to estimate $N$ of PTMCs, in agreement with the LCM.

\section{Results and discussion}

GaSe and InSe samples are prepared using a micromanipulation setup placed inside an Ar filled glovebox to prevent the air-induced degradation. Bulk PTMC crystals are mechanically exfoliated onto a $200 \mathrm{~nm}$ layer of poly-(propylene carbonate) (PPC), spin coated onto a Si wafer and identified using optical microscopy. ${ }^{20}$ Selected PTMC flakes are then picked up with thin hBN layers residing on a polymer membrane using a dry peel transfer technique. ${ }^{19}$ The resulting PTMC/hBN stack is then transferred onto another hBN crystal exfoliated onto a $\mathrm{Si} / \mathrm{SiO}_{2}$ substrate, to achieve full encapsulation. The verification of the PTMC thicknesses is performed using atomic force microscopy (AFM).

Raman measurements are carried out in backscattered geometry at room temperature using Renishaw, Horiba LabRam HR Evolution, Horiba XploRa and Horiba FHR1000 systems equipped with interference or Bragg grating filters, with a cut-off at $5 \mathrm{~cm}^{-1}$. The Raman spectra are taken at $633 \mathrm{~nm}(1.96 \mathrm{eV}), 532 \mathrm{~nm}(2.33$ $\mathrm{eV}), 515 \mathrm{~nm}(2.41 \mathrm{eV}), 488 \mathrm{~nm}(2.54 \mathrm{eV})$, and $457 \mathrm{~nm}(2.71 \mathrm{eV})$. The laser is focused by a $100 \times$ objective. The laser power on the sample is kept at $\sim 150 \mu \mathrm{W}$ during all measurements, to avoid damage and local heating. The collected signal is dispersed with 1800 and 2400 grooves $\mathrm{mm}^{-1}$ gratings and detected with a CCD camera. For each measurement, the spectrum from an area near each studied layer is subtracted, to remove the Si substrate background. The background spectra are also used for calibration of the data from different setups, using the Si Raman mode at $520.7 \mathrm{~cm}^{-1} .^{21}$

Fig. 2 plots the polarization-resolved Raman spectra of three layer (3L) GaSe and InSe, accompanied by the corresponding atomic displacement of the phonon modes. 3L GaSe has two out-of-plane phonon modes, $A_{1}^{\prime}(1)$ at $\sim 135 \mathrm{~cm}^{-1}$ and $\mathrm{A}_{1}^{\prime}(2)$ at $\sim 308 \mathrm{~cm}^{-1}$, and one in-plane $\mathrm{E}^{\prime \prime}(2)$ mode at $\sim 214 \mathrm{~cm}^{-1}$. 3L InSe has 3 outof-plane modes: $\mathrm{A}_{1}^{\prime}(1)$ at $\sim 115 \mathrm{~cm}^{-1}, \mathrm{~A}_{2}^{\prime \prime}(1)$ at $\sim 200 \mathrm{~cm}^{-1}$ and $\mathrm{A}_{1}^{\prime}(2)$ at $\sim 228 \mathrm{~cm}^{-1}$, and one in-plane $E^{\prime \prime}(2)$ mode at $\sim 178 \mathrm{~cm}^{-1}$. The peaks are classified according to their irreducible representation in the symmetry group in the $1 \mathrm{~L}$ phase $D_{3 \mathrm{~h}}$, and additionally numbered due to their increased Raman shift. This notation is consistent with earlier works on bulk $\varepsilon$-GaSe, $\gamma$-InSe. ${ }^{22-25}$ 

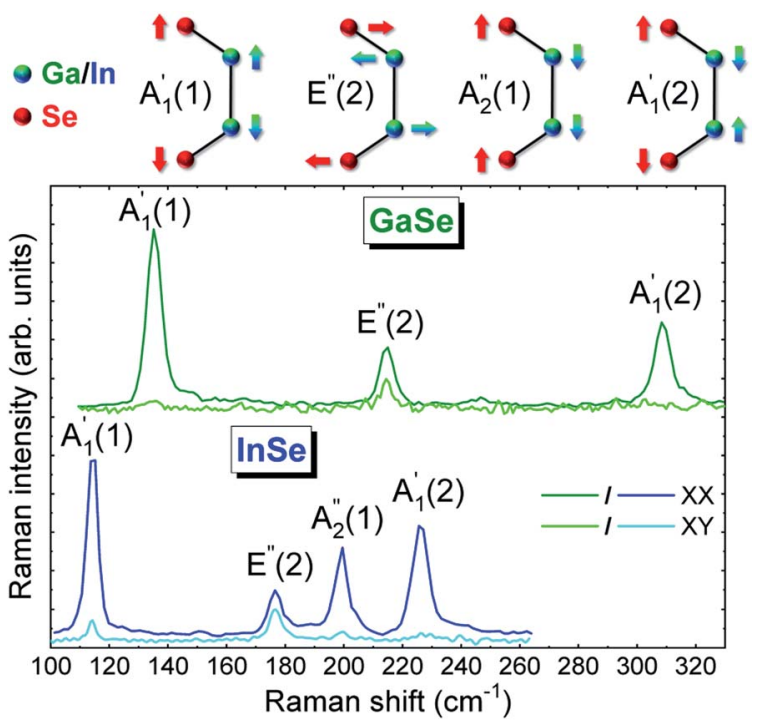

Fig. 2 Atomic displacements of phonon modes and polarization-resolved $514.5 \mathrm{~nm}$ Raman spectra of $3 \mathrm{~L}$ GaSe (green/bright green) and InSe (blue/bright blue) in XX/XY configurations.

To confirm their symmetries, the Raman spectra are recorded at $488 \mathrm{~nm}$ for 2 polarizations: parallel $-\mathrm{XX}$ and cross-polarized $-\mathrm{XY}$. For both materials, all peaks are observed in XX, while only $\mathrm{E}^{\prime \prime}(2)$ modes are seen in $\mathrm{XY}$, as expected due to the rotational symmetry in these crystals, which implies that A-type modes are only active in parallel polarization, while E-type modes are observable in both (see ESI $\dagger$ for details).

In order to reveal the evolution of the Raman spectra with $N$, crystals with multiple terraces with lateral size $>1 \mu \mathrm{m}$ are studied. The unpolarized Raman spectra, presented in Fig. $3 \mathrm{a}$ and $\mathrm{b}$, show the optical phonon modes down to $1 \mathrm{~L}$.

For GaSe, $\mathrm{A}_{1}^{\prime}(1)$ and $\mathrm{A}_{1}^{\prime}(2)$ red- and blue-shift, respectively, with decreasing $N$, while $\mathrm{E}^{\prime \prime}(2)$ is barely affected by $N$. For bulk, a double peak is observed, comprising $\mathrm{E}^{\prime \prime}(2)$ at $\sim 211 \mathrm{~cm}^{-1}$ and $\mathrm{E}^{\prime}(2)$ at $\sim 216 \mathrm{~cm}^{-1}$, consistent with first principles calculations (see ESI $\dagger$ ), and previous Raman studies of bulk crystals. ${ }^{22,23}$ The $\mathrm{E}^{\prime}(2)$ mode corresponds to an in-plane phonon, where Ga or Se atoms vibrate in-phase, while an out-of-phase vibration occurs between $\mathrm{Ga}$ and Se pairs.

For InSe, Fig. 3b, we observe a similar red- and blue-shift of $A_{1}^{\prime}(1)$ and $A_{1}^{\prime}(2)$ with decreasing $N$, and no significant shift of $\mathrm{E}^{\prime \prime}(2)$, while $\mathrm{A}_{2}^{\prime \prime}(1)$ blueshifts like $\mathrm{A}_{1}^{\prime}(2)$. For $16 \mathrm{~L}$ and bulk, an additional peak is seen at $\sim 210 \mathrm{~cm}^{-1}$, visible under $\mathrm{XY}$ polarization, suggesting it to be an E-type vibration. As for ref. 26-28, we assign it to an $E^{\prime}(2)$ mode, similar to GaSe bulk. The observation of $A_{2}^{\prime \prime}(1)$ in the Raman spectrum of InSe bulk was previously ascribed to resonance with the B excitonic transition. ${ }^{25}$ Ref. 3 showed that the B energy is affected by $N$, changing from $\sim 2.4 \mathrm{eV}$ in bulk to $\sim 2.9 \mathrm{eV}$ for $1 \mathrm{~L}$. For a constant excitation of $2.54 \mathrm{eV}$ as used in Fig. 3, the resonance is strongest for intermediate thicknesses, with the largest $\mathrm{A}_{2}^{\prime \prime}(1)$ intensity for $5 \mathrm{~L}$, Fig. $3 \mathrm{~b}$. 


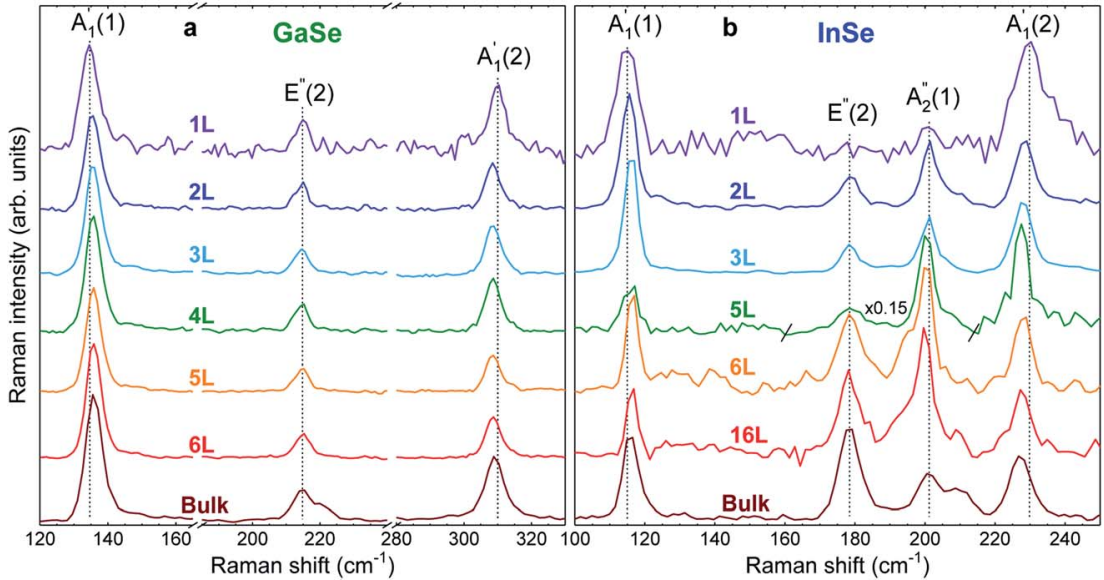

Fig. 3 Unpolarized Raman spectra as a function of $N$ for (a) GaSe and (b) InSe measured at $488 \mathrm{~nm}$. Dashed lines show peak positions for $1 \mathrm{~L}$.

The evolution of peaks' positions with $N$, extracted using Lorentzian fits, for both materials is shown in Fig. $4 \mathrm{a}$ and $\mathrm{b}$. Previous publications reported only one phonon mode in thin crystals down to $1 \mathrm{~L}$, i.e. $\mathrm{A}_{1}^{\prime}(2)$ for $\mathrm{GaSe}^{15}$ and $\mathrm{A}_{1}^{\prime}(1)$ for InSe, ${ }^{16}$ likely due to the lack of encapsulation, leading to crystal degradation. ${ }^{29}$ The reported $N$ evolution of $\mathrm{A}_{1}^{\prime}(2)$ in $\mathrm{GaSe}^{15}$ is opposite (a redshift with decreasing $N$ ) to our observations, which may be due to an overlap of the $A_{1}^{\prime}(2)$ mode with Si modes $\sim 300 \mathrm{~cm}^{-1}$ in ref. 20 . In InSe, a similar redshift of $A_{1}^{\prime}(1)$ was previously reportedobserved down to $2 \mathrm{~L} .{ }^{16}$ However, the Raman signal of $1 \mathrm{~L}$ was not detected, and only $\mathrm{A}_{1}^{\prime}(1)$ was visible in $2 \mathrm{~L}$.

We further investigate the $N$ dependence of the difference in peaks' positions between $\mathrm{A}_{1}^{\prime}(2)$ and $\mathrm{A}_{1}^{\prime}(1)$, i.e. $\Delta=\mathrm{A}_{1}^{\prime}(2)-\mathrm{A}_{1}^{\prime}(1)$. According to our DFT calculations presented in the ESI, $\uparrow$ a gradual rise in $\Delta$ with decreasing $N$ is predicted for both materials, Fig. 4c. This matches our experimental results for InSe, where $\Delta$ is
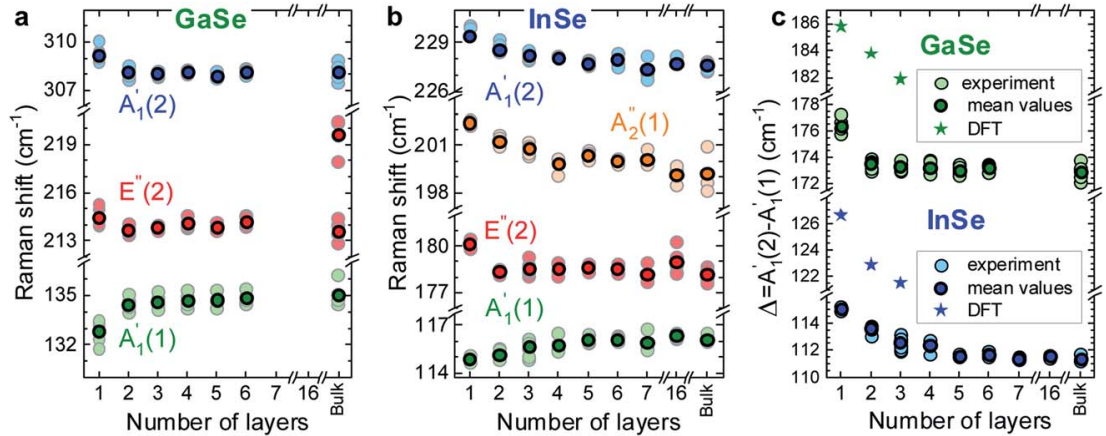

Fig. $4 N$-dependent Raman shifts for (a) GaSe and (b) InSe. (c) $N$ dependence of $\Delta=\mathrm{A}_{1}^{\prime}(2)-\mathrm{A}_{1}^{\prime}(1)$. DFT calculations are indicated by star symbols. The dark color symbols correspond to mean values for the dataset shown in light colors measured at 633, 533, 514.5, 488 and $457 \mathrm{~nm}$. 
gradually increasing from $5 \mathrm{~L}$ to $1 \mathrm{~L}$, with a total blueshift $\sim 3.5 \mathrm{~cm}^{-1}$. A more abrupt change is observed in GaSe, where $\Delta$ only changes noticeably in $1 \mathrm{~L}$ by $\sim 3 \mathrm{~cm}^{-1}$. $\Delta$ in both materials is enough to be used for assessing $N$ in few layer (FL) In Se and 1L-GaSe, similar to what done for TMDs. ${ }^{29,30}$

Fig. 5 plots the low frequency Raman spectra of InSe and GaSe flakes. Fig. 5a and $b$ show 3 types of Raman peaks for both materials. Two of them are due to the vibrations in the GaSe and InSe layers, while the peak at $\sim 52 \mathrm{~cm}^{-1}$, indicated by *, is the $\mathrm{C}$ peak of the encapsulating hBN.

The evolution of C and LB modes can be described using a LCM, where the layers are represented as one mass connected to the next layer by a spring with a coupling constant. $\operatorname{Pos}(\mathrm{C})$ and $\operatorname{Pos}(\mathrm{LBM})$ can then be written as: $:^{11-13}$

$$
\begin{gathered}
\operatorname{Pos}\left(\mathrm{C}_{N, N-i}\right)=\frac{1}{\pi c} \sqrt{\frac{\alpha_{\|}}{\mu}} \sin \left[\frac{i \pi}{2 N}\right] \\
\operatorname{Pos}\left(\mathrm{LBM}_{N, N-i}\right)=\frac{1}{\pi c} \sqrt{\frac{\alpha_{\perp}}{\mu}} \sin \left[\frac{i \pi}{2 N}\right]
\end{gathered}
$$

where $\alpha_{\|}$and $\alpha_{\perp}$ are force constants per unit area for the shear motion, denoted $\|$, and the layer breathing motion, denoted $\perp$, between two layers. $\mu$ is the $1 \mathrm{~L}$ mass per unit area. $i=1,2, \ldots N-1$. Multiplying $\alpha_{\|}$and $\alpha_{\perp}$ by the unit cell area gives the interlayer force constants, $K .^{11-13}$

We also use DFT within the local density approximation (LDA) to calculate the vibrational frequencies, as detailed in the ESI. $\dagger$ To compute the C and LBM frequencies, we rely on the frozen phonon approximation to calculate the corresponding force constant between neighbouring layers. The interlayer force constant remains the same, within $1 \%$, when increasing $N$ from 2 to 3 . Therefore, the LCM approach can be applied to $\mathrm{C}$ modes under the assumption that independent force constants describe the coupling between neighbouring layers. Such an approximation was previously applied to $\varepsilon$-GaSe. ${ }^{31}$ We obtain the interlayer
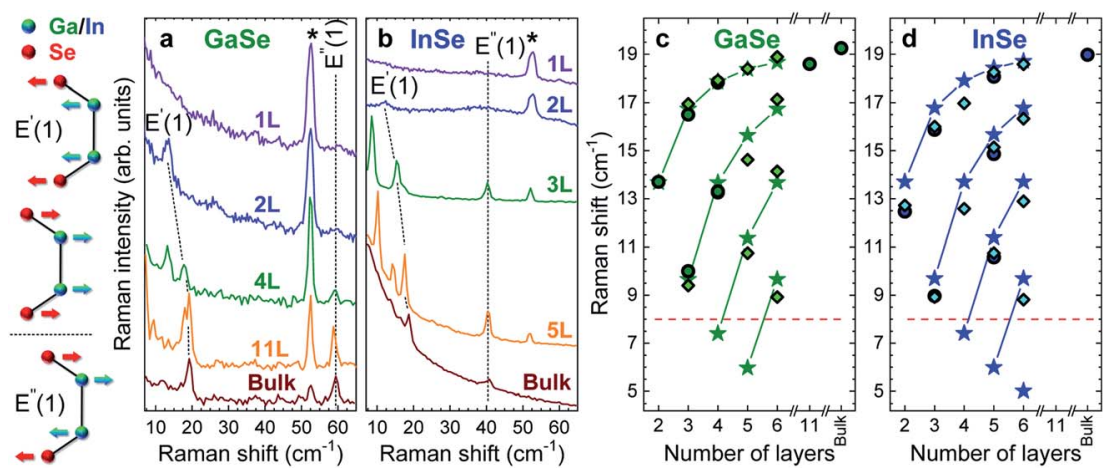

Fig. 5 Atomic displacements of phonon modes and unpolarized $514.5 \mathrm{~nm}$ low-frequency Raman spectra as a function of $N$ for (a) GaSe and (b) InSe. Dashed lines are guides to the eye. ( $c$ and d) $N$ dependence of $C$ modes of (c) GaSe and (d) InSe. Circles and diamonds denote experimental results. Stars are DFT calculations. Dashed red horizontal lines represent the minimal Raman shift $\sim 8 \mathrm{~cm}^{-1}$ detectable in our experiments. 
force constants: for C modes, $K^{\mathrm{GaSe}}=102 \mathrm{meV} \AA^{-2}$ and $K^{\mathrm{InSe}}=134 \mathrm{meV} \AA^{-2}$; for LBMs, $K^{\mathrm{GaSe}}=323 \mathrm{meV} \AA^{-2}$ and $K^{\mathrm{InSe}}=401 \mathrm{meV}^{-2}$.

In our experiments, we only observe $\mathrm{C}$ modes. Fig. $5 \mathrm{c}$ and $\mathrm{d}$ plot experimental and calculated $\operatorname{Pos}(\mathrm{C}) .^{34}$ The results are in good agreement, both concerning frequencies and number of branches for a given $N$. By fitting the measured Pos(C) as function of $N$ with eqn (1), we get $\alpha_{\|} \sim 14.4 \times 10^{18} \mathrm{~N} \mathrm{~m}^{-3}$ for InSe and $\sim 13.3 \times$ $10^{18} \mathrm{~N} \mathrm{~m}^{-3}$ for GaSe. We use the experimental values for the lattice constants $a_{\mathrm{InSe}}^{\exp }=4.002 \AA$ (ref. 32) and $a_{\mathrm{GaSe}}^{\exp }=3.755 \AA$ (ref. 33) to extract the unit area. Using the LDA lattice parameters $a_{\text {GaSe }}=3.6678 \AA$ and $a_{\text {Inse }}=3.9103 \AA$ and computing the unit cell areas, we can extract $\alpha_{\|}$from the theoretical interlayer force constants $K$ for InSe and GaSe by dividing $K$ by the unit cell area. We get $16 \times 10^{18} \mathrm{~N} \mathrm{~m}^{-3}$ for InSe and $14 \times 10^{18} \mathrm{~N} \mathrm{~m}^{-3}$ for GaSe, in good agreement with the experimentally determined values.

\section{Conclusions}

We performed a Raman spectroscopic investigation of InSe and GaSe flakes of varying number of layers from bulk down to a monolayer, for samples encapsulated in hBN. We determined the thickness-dependent evolution of both intralayer and interlayer modes, and compared the results with first principles calculations. We confirmed that Raman spectroscopy can be used to estimate the number of layers of PTMCs.

\section{Conflicts of interest}

There are no conflicts to declare.

\section{Acknowledgements}

We acknowledge support from the National Science Centre, Poland (grants no. 2017/24/C/ST3/00119, 2017/27/B/ST3/00205), EPSRC ARCHER RAP grant (e547), EU Graphene Flagship and Quantum Technology Flagships, The Scientific and Technological Research Council of Turkey (TUBITAK) through BIDEB-2219 programme (2018-1), EPSRC CDT Graphene-NOWNANO and Graphene Technology, EPSRC Doctoral Prize Fellowship, Royal Society Research Fellowship, Samsung Advanced Institute of Technology (SAIT), ERC Grant Hetero2D, EPSRC Grants EP/ K01711X/1, EP/K017144/1, EP/N010345/1 and EP/L016057/1.

\section{References}

1 A. C. Ferrari, et al., Nanoscale, 2015, 7, 4598.

2 W. Choi, N. Choudhary, G. H. Han, J. Park, D. Akinwande and Y. H. Lee, Mater. Today, 2017, 20, 116.

3 G. W. Mudd, et al., Sci. Rep., 2016, 6, 39619.

4 D. A. Bandurin, et al., Nat. Nanotechnol., 2017, 12, 223.

5 D. J. Terry, et al., 2D Materials, 2018, 5, 041009.

6 M. J. Hamer, et al., ACS Nano, 2019, 13, 2136.

7 M. Hamer, et al., Nano Lett., 2018, 18, 3950.

8 N. Ubrig, et al., 2019, arXiv:1912.10345. 
9 S. Jandl and C. Carlone, Solid State Commun., 1978, 25, 5.

10 A. C. Ferrari, et al., Phys. Rev. Lett., 2006, 97, 187401.

11 A. C. Ferrari and D. M. Basko, Nat. Nanotechnol., 2013, 8, 235.

12 P. H. Tan, et al., Nat. Mater., 2012, 11, 294.

13 X. Zhang, et al., Phys. Rev. B: Condens. Matter Mater. Phys., 2013, 87, 115413.

14 D. J. Late, et al., Adv. Mater., 2012, 24, 3549.

15 D. J. Late, et al., Adv. Funct. Mater., 2012, 22, 1894.

16 Z. Chen, K. Gacem, M. Boukhicha, J. Biscaras and A. Shukla, Nanotechnology, 2013, 24, 415708.

17 L. Quan, et al., J. Mater. Chem. C, 2015, 3, 11129.

18 Y. Li, et al., 2D Materials, 2018, 5, 021002.

19 Y. Cao, et al., Nano Lett., 2015, 15, 4914.

20 C. Casiraghi, et al., Nano Lett., 2007, 7, 2711.

21 P. A. Temple and C. E. Hathaway, Phys. Rev. B: Solid State, 1973, 7, 3685.

22 N. Kuroda and Y. Nishina, Solid State Commun., 1980, 34, 481.

23 N. M. Gasanly, A. Aydınlı, H. Özkan and C. Kocabaş, Mater. Res. Bull., 2002, 37, 169.

24 R. M. Hoff, J. C. Irwin and R. M. A. Lieth, Can. J. Phys., 1975, 53, 1606.

25 N. Kuroda and Y. Nishina, Solid State Commun., 1978, 28, 439.

26 N. M. Gasanly, B. M. Yavadov, V. I. Tagirov and E. A. Vinogradov, Phys. Status Solidi B, 1978, 89, K43.

27 S. Ashokan, K. P. Jain, M. Balkanski and C. Julien, Phys. Rev. B: Condens. Matter Mater. Phys., 1991, 44, 11133.

28 I. H. Choi and P. Y. Yu, J. Appl. Phys., 2003, 93, 4673.

29 O. Del Pozo-Zamudio, et al., 2015, arXiv:1506.05619.

30 C. Lee, et al., ACS Nano, 2010, 4, 2695.

31 R. Longuinhos and J. Ribeiro-Soares, Phys. Chem. Chem. Phys., 2016, 18, 25401.

32 J. Rigoult, A. Rimsky and A. Kuhn, Acta Crystallogr., Sect. B: Struct. Crystallogr. Cryst. Chem., 1980, 36, 916.

33 F. Jellinek and H. Hahn, Z. Naturforsch., B, 1961, 16b, 713.

34 G. Pizzi, S. Milana, A. C. Ferrari, N. Marzari and M. Gibertini, 2020, arXiv:2011.14681. 